\title{
Giant Cell Tumor Arising from Anterior Arc of First Rib: A Rare Presentation
}

\author{
Pandey $S,{ }^{1}$ Thakur B, ${ }^{1}$ Chhetri $\mathrm{P}^{1}$ \\ 'Department of Orthopedics, B.P. Koirala Memorial Cancer Hospital, Bharatpur, Nepal.
}

\begin{abstract}
We report a case of Giant Cell Tumor (GCT) arising from anterior arc of first rib along with literature review which was treated with excision of rib through its normal margin along with tumor mass. GCT of rib is rare and if found mostly arises from the posterior aspect. However, GCT should be considered as differential diagnosis when bony mass is present in the anterior arc of rib. It poses surgical challenge for excision because of the delicate and vital neurovascular structures passing above it between clavicle and first rib.
\end{abstract}

Keywords: first rib, giant cell tumor

\section{INTRODUCTION}

Giant Cell Tumor (GCT) is found most often at the end of skeletally mature long bones essentially arising from epiphysis. The lesion is of uncertain cell origin and comprises of $5 \%$ of all bony neoplasm according to Mayo clinic series. The rib is rare site for GCT with an incidence of less than $1 \%$ of all GCT. ${ }^{1}$ The cases reported earlier in the literature, most were located in the posterior arc i.e. head and tubercle of rib. ${ }^{2,3} \mathrm{We}$ are reporting a rare presentation of GCT arising from anterior arc of first rib because of its rarity and challenge for its surgical dissection from adjacent vital neurovascular structures while excision of tumor mass.

\section{CASE REPORT}

A 22-year-old lady presented to Orthopedic OPD with progressively increasing pain and swelling over right anterior chest wall just below the clavicle for six month. She did not have any constitutional symptoms.
On examination her general condition was fair and respiratory, cardiovascular system, abdomen and spine were within normal limit. Local examination revealed reddish discoloration of overlying skin with diffuse fullness over first rib region below the clavicle on anterior chest wall. Overlying skin was warm. There was ill defined, diffuse, tender, hard mass arising from anterior arc of first rib. Plain X-ray chest PA view showed well defined, multiloculated, expansile mass, classical soap bubble appearance as seen in GCT, arising from anterior arc of first rib. CT scan of the chest showed expansile lytic lesion involving anterior end of right rib with cortical thinning without periosteal reaction. There was no soft tissue involvement.

FNAC from the lesion revealed features suggestive of GCT. Haemogram, random blood sugar, urea, creatinine, serum calcium, alkaline phosphatase and liver function test were not significant. She was treated with excision of first rib through its normal margin at angle of rib along with tumor mass under general anesthesia in

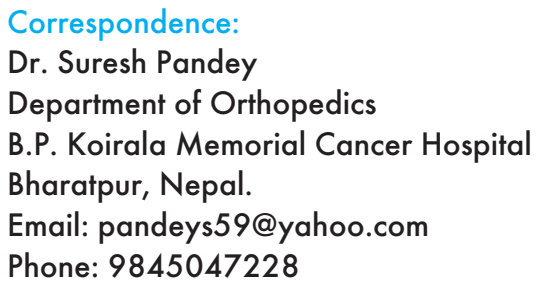


supine position. Supraclavicular approach was used and careful dissection was done to visualize, isolate and protect brachial plexus, subclavian artery and vein, supraclavicular nerve and vessels and phrenic nerve. The resected mass was $8 \mathrm{~cm} \times 4 \mathrm{~cm}$ in size and cut section showed fleshy friable mass with haemorrhage. Histopathological examination revealed spindle shaped mononuclear cells with uniformaly interspersed abundant multinucleated giant cells. The clinicoradiological diagnosis of GCT was confirmed in biopsy. There was no neurovascular deficit in post operative phase and patient is asymptomatic and without local recurrence or pulmonary metastasis in 12 months follow up.

\section{DISCUSSION}

GCT is less common variety of bony neoplasm comprising of only four to five percent of all bony neoplasm. It arises from epiphyses of long bone, most commonly lower end of femur, upper end of tibia and lower end of radius in order of its frequency. Other sites are uncommon, rib is its very uncommon sites and only few cases have been reported to originate from anterior arc of rib. ${ }^{4,5} \mathrm{GCT}$ arising from anterior arc of first rib is still rare and poses diagnostic difficulty because of its rarity and surgical challenge because of its adjacent vital neurovascular structures. Though in our case the presentation was of classical type, it doesn't cause diagnostic difficulties. Treat with excision of the tumor mass through its normal margin is surgically challenging because of its relation with subclavian vessels, brachial plexus, phrenic nerve and limited access due to clavicle lying anteriorly.

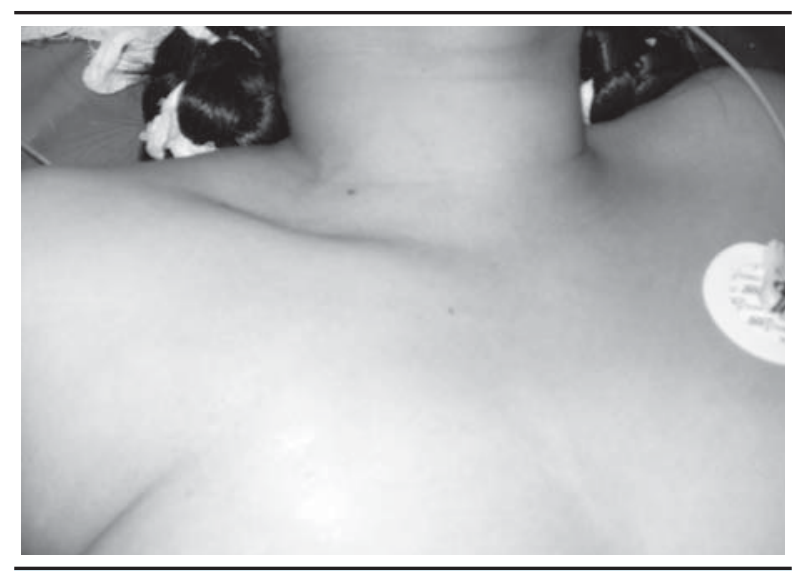

Figure 1 . Swelling on $\mathbf{1}^{\text {st }}$ rib region

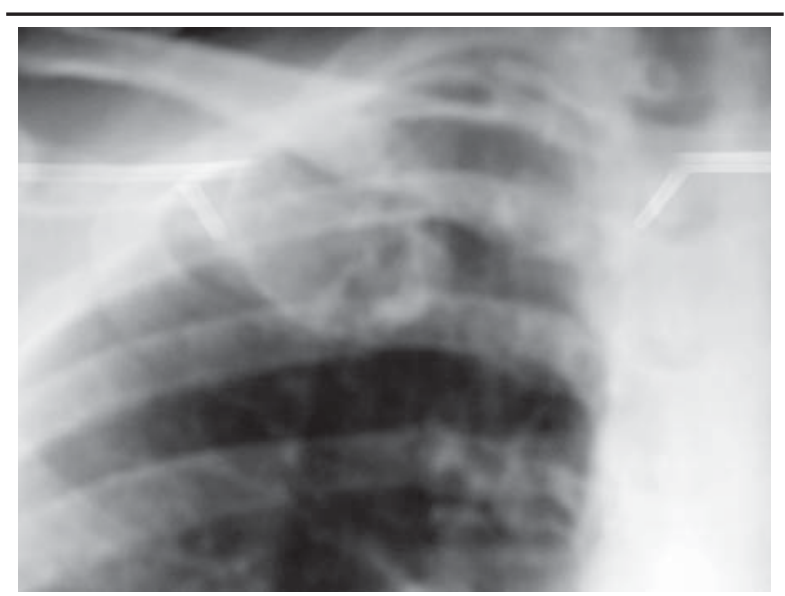

Figure 2. X-Ray chest showing mass arising from $1^{\text {st }}$ rib anteriorly

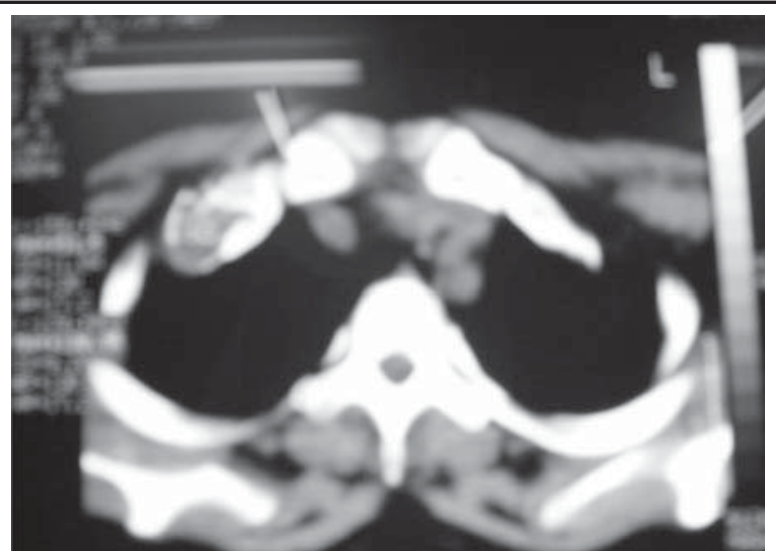

Figure 3. CT scan chest showing mass arising from anterior arc of $1^{\text {st }}$ rib

\section{REFERENCES}

1. Hanna RM, Kyriakos M, Quinn SF. Case report 757: giant cell tumor of rib. Skeletal Radiol. 1992;21:482-8.

2. Bonnet D, Barrier JR, Zimmermann JM, Eskandari J, Martet G, Robert JL. Giant Cell Tumors of ribs. Apropos of case. Rev pneumol Clin. 1992;48:79-85.

3. Tanaka F, Wada H, Mizuno H, Hitomi S. A case of giant cell tumor of the rib with magnetic resonance imaging. Eur J Cardiothorac Surg. 1996;10:214-6.

4. Gupta V, Mittal R. Giant cell tumor of rib: rare location on the anterior aspect. Arch Orthop Trauma Surg. 2000:120:231-2.

5. Brenner P, Warbanow FK, Krause-Bergmann A, Berger A. Rare differential diagnosis of breast tumor. Giant cell tumor of the ribs. Langenbecks Arch Chir. 1997:382:64-8. 